Accepted for publication in Waste Management Published in November, 2014

DOI: 10.1016/j.wasman.2014.05.006.

This accepted author manuscript is copyrighted and published by Elsevier. It is posted here by agreement between Elsevier and MTA. The definitive version of the text was subsequently published in [Waste Management, volume 34, issue 11, November 2014, DOI: 10.1016/j.wasman.2014.05.006]. Available under license CC-BY-NC-ND.

\title{
Alternative polymer separation technology by centrifugal force in a melted state
}

\author{
Károly Dobrovszky, Ferenc Ronkay* \\ Department of Polymer Engineering, Faculty of Mechanical Engineering, Budapest \\ University of Technology and Economics, \\ H-1111 Budapest, Müegyetem rkp. 3, Hungary \\ * Corresponding author. Tel.: +36 1 463-2462. \\ E-mail address: ronkay@pt.bme.hu (F. Ronkay).
}

\begin{abstract}
:
In order to upgrade polymer waste during recycling, separation should take place at high purity. The present research was aimed to develop a novel, alternative separation opportunity, where the polymer fractions were separated by centrifugal force in melted state. The efficiency of the constructed separation equipment was verified by two immiscible plastics (polyethylene terephthalate, PET; low density polyethylene, LDPE), which have a high difference of density, and of which large quantities can also be found in the municipal solid waste. The results show that the developed equipment is suitable not only for separating dry blended mixtures of PET/LDPE into pure components again, but also for separating prefabricated polymer blends. By this process it becomes possible to recover pure polymer substances from multi-component products during the recycling process. The adequacy of results was verified by differential scanning calorimetry (DSC) measurement as well as optical microscopy and Raman spectroscopy.
\end{abstract}

Keywords: polymer separation; centrifugal force; blend separation; melted state separation; polymer waste.

\section{Introduction}

Several options of separating materials from each other have gained new impulses of development, due to their adequacy for a more pronounced way of recycling. In the past three decades, the use of polymers has been increased continuously. The production of plastic materials has been increasing by about 5\% annually. As a consequence, the polymer waste stream has been rising too, especially that of municipal solid waste. By processing only lowquality products it can be realized if the whole waste stream is used at the same time, which is 
called 'downcycling'. More than half of polymer materials are discarded every year despite their durability, so it is necessary to separate and recycle polymers because of social pressure and environmental aspects (Alter, 2005; Bezati et al., 2011; Wu et al., 2013). Thus, it would be desirable to establish a new, alternative separation method, which enables the sorting of assorted waste products into individual substances from the waste stream possible in one step, regardless of the materials (Zhang et al., 2012). The processing temperature, density, and molecular and physical characteristics are different for all polymer materials, so they are usually not compatible with each other. Therefore, it would be desirable to separate the waste component with high effectiveness. Separating the fine purity of the waste component is the most important precondition for producing good quality products. When the polymers are washed and separated from each other, and further additives are added at manufacturing - such as fibre reinforcement or chemical agents - it could be possible to produce higher quality products than virgin materials. This process is called 'upgrading' or 'upcycling' (Vermeulen et al., 2011).

The management of post-consumer plastic waste is complicated since the greater part of it is placed into communal rubbish. The separated polymer waste from post-consumers is typically: (i) polyethylene terephthalate (PET) from soft drink bottles; (ii) polypropylene (PP); (iii) polyethylene (PE) with high (HDPE) or low density (LDPE) from packaging and bottles; (iv) polystyrene (PS) from drinking cups and pots; and (v) polyvinyl chloride (PVC) from pipes and interior furnishings (Bezati et al., 2011; Pongstabodee et al., 2008). Given that the content of the post-consumed polymer waste is heterogeneous it is necessary to separate it into pure polymer component. The recycling process can be more complicated if the polymers contain fibre reinforcement or other additives, or if the material is foamed. These components modify the density, thus it is not possible to assign a fixed density value to the polymer. A density range can only be established where the individual component can be found. In addition, splitting polymer blends into neat substances is not solved yet with the current sorting equipment. These polymer blend parts are present as contaminants in the separation process, and impair the quality of the recycling process.

As is currently known, it is difficult to achieve the required purity of mixed polymer waste in just one step. Indeed, the grade of PP and PE from waste should be higher than 97\%, in order to be suitable for manufacturing at high level (Bakker et al., 2009). Multi-step separation methods are usually applied to industrial processes. By simple, common density-based separation technologies, it is difficult to separate mixed plastic waste constituents from each other, when there are slight differences in density, such as PET and PVC mixtures (Burat et al., 2009; Sadat-Shojai and Bakhshandeh, 2011; Takoungsakdakun and Pongstabodee, 2007).

The simplest separation method is hand-sorting. The pickers separate the materials from each other, while the hazardous waste, metal parts and non-polymer waste is removed. The disadvantage of this process is the low output and errors made by humans, so it is suitable as an option of pre-sorting (Super et al., 1993). The same principle applies to optical sorting, which can be automated, and is another way of pre-sorting, whereby an optical light, infrared or Xray source can be used. The absorbed light can be exploited in this way and is reflected differently by the polymers owing to the specific state of electrons which characterize every 
polymer substance (Arvanitoyannis and Bosnea, 2001; Bodzay et al., 2009; Serranti et al., 2011). With optical methods, polymers can only be sorted by colour, except for black painted materials. Near-infrared technology is also unsuitable for dark objects. However, with midinfrared ranges dark-coloured polymers can be identified. X-ray spectroscopy is only used to isolate PVC materials (Alter, 2005; Zhang et al., 2012). Another possibility for pre-separation is the density-based separation method. In this case, separation is feasible with dry particles, using air classifiers, or water-based solutions or suspensions as separating media. The particles sink or float in the fluid depending on the force of buoyancy (Gent et al., 2011). One simple way of separation is the water separation method as an intermediate density medium, where the municipal solid plastic waste can be separated into less than $1 \mathrm{~g} / \mathrm{cm}^{3}$ polyolefin and other polymers of higher density.

Standard industrial solutions for polymer waste separation of individual components are: (i) integrated spectral imaging analysis (Bodzay et al., 2009; Serranti et al., 2011); (ii) gravity separation of density-based of polymers; (iii) froth flotation; (iv) electrostatic separation; and (v) magnetic separation (Bakker et al., 2009; Burat et al., 2009; Carvalho et al., 2007; Pongstabodee et al., 2008; Sadat-Shojai and Bakhshandeh, 2011; Takoungsakdakun and Pongstabodee, 2007). The literature also mentions centrifugal force separation (Gent et al., 2009), separation in a melted state and chemical solving (Bakker et al., 2009), which are used rather within laboratory conditions.

Surveying separation by spectral signs, Gondal and Siddiqui (2007) found that laser-induced breakdown spectroscopy is suitable for detecting PE, PP and ABS molecules. Fourier transform infrared spectroscopy (FTIR) and Raman spectroscopy are widely used for detecting the purity of the separated final polymer waste, after the measurement of the spectral signals for pure substances (Fávaro et al., 2013; Giancola et al., 2012; Ramesh et al., 2007; Serranti et al., 2011; Vajna et al., 2012).

Triboelectrostatic separation can be a solution for post-consumer waste management. With different materials touching each other they have a different charge in opposite polarities. It is well-known that two particles with different surface properties, in contact with each other, may be charged, and electron transfer happens until equality of the Fermi levels of the touching materials. This phenomenon is called triboelectric charging effect or tribocharging (Matsusaka et al., 2010). After this step, charged particles are fed into an electric field to separate the fractions into homogeneous parts, because the trajectories of particles are deflected, according to the polarities and the quantity of the charge.

Density-based separation can be accomplished either with dry particles and separation medium, or by water-based suspensions. The division of polymers is based on the effects of sinking or floating of the particles in the separation medium. The process is driven by the force of buoyancy (Gent et al., 2009). Super et al. (1993) used a mixture of near-supercritical fluids sulphur hexafluoride $\left(\mathrm{SF}_{6}\right)$ and carbon dioxide $\left(\mathrm{CO}_{2}\right)$ - to separate post-consumer plastic waste. The density of the separation media was changeable, depending on the ratio of the gases. The results showed a pollution grade of 3 to $5 \%$ in the neat separated polyolefin fractions, and less than $1 \%$ of cross-contamination in PET/PVC mixtures. A wet shaking table is a wet waste 
separation method, where the relative movement responds to gravity and other forces. To separate plastic waste from one another a minimum difference of density of the materials is needed, which has been determined by Wills (1997). Carvalho et al. (2007) investigated the efficiency of wet shaking table separation of PS/PET/PVC mixtures. The results showed that it was possible to separate whole amounts of PC from a PET/PVC waste stream in one step. They found if a higher grade of PET purity was needed a lower recovery was obtained, and vice versa.

The separation of plastic mixture is not possible because of the slight differences in density. Therefore, it is necessary to also use a frothing and wetting agent, to achieve acceptable purity of the substances. Pongstabodee et al. (2008) developed a three-stage sink-float method with selective flotation, to separate post-consumer plastic waste into pure fragments. Burat et al. (2009) used also froth flotation to separate virgin and recycled PET and PVC. They found that strong alkaline solutions are able to destroy the hydrophobicity of PET more than PVC. So PET was rendered to hydrophilic, while the PVC remained in a hydrophobic state. It can be concluded that separating post-consumer waste is more difficult because of the degradation and dirtiness of the waste.

Bakker et al. (2009) developed an alternative method of separating polyolefin fractions. They used inverse magnetic density separator (IMDS), where a lower apparent density than water could be achieved by the separation medium, because of the combination of the gradient magnetic field and the magnetic liquids, where the latter contains nanoscale ferrite particles. So it is possible to make the suspension artificially lighter or heavier. They used a splitter, with which the height of the separation line could be adjusted in the liquid after the mixing zone. PE particles were flown under, while PP flown over the splitter (Fig. 1). The results of the research of Bakker showed a $98 \%$ purity and $72 \%$ recovery rate for PP. A higher grade of purity of PE can be achieved with a second splitter to separate fractions into three groups.

Taking advantage of the centrifugal force field is another opportunity to separate materials, which is exploited in many other areas. Centrifugation is a density-based separation technology, based on the density differences of the particles. Centrifugal separators can exert huge forces on the liquid and particles, so the contamination can be isolated from the other fraction or the fractions can be separated from each other. Eq. (1) shows that the separation force depends on the density of the particle and the spinning speed, in 1D case at small Reynolds number $(R e<$ $1)$.

$$
F_{c f}=m \cdot a_{c f}=\rho_{a} \cdot V_{a} \cdot r \cdot\left(2 \cdot \pi \cdot n_{c, m} \cdot 60\right)^{2}
$$

where $F_{c f}$ is the fictitious centrifugal force, $m$ is the mass of the particles, $a_{c f}$ is the centripetal acceleration, $r$ is the distance between the axis of rotation and the particle, $\mathrm{n}_{\mathrm{c}, \mathrm{m}}$ is the speed of the equipment in minutes, $\rho_{\mathrm{a}}$ and $V_{\mathrm{a}}$ are the density and the volume of the particle $a$, respectively.

This procedure is used for the fabrication of biodegradable polymer fibres (Wang et al., 2011), in mineral processing at classification and separation, dewatering or waste-water treatment (Batalović, 2011), producing highly integrated biodiesel fuels (Kraai et al., 2009) and particle 
classification (Yamamoto et al., 2009). This technology is also significant as it is used in the field of medical technology (Kim et al., 2013) and in the food industry (Strixner and Kulozik, 2013). The favourable properties of the centrifugation could be exploited for polymer separation too.

The aim of the present paper is the presentation of a novel separation device, which is suitable for the separation of high purity, different virgin or recycled polymers. The device is also applied to separate prefabricated polymer blends into their former base components. The developed equipment utilizes centrifugal force to separate the fractions in a melted state. Consequently, we selected two plastics (PET and LDPE), which also appeared in large amounts in post-consumer plastic waste, to prove our system's operability and the effectiveness of separation.

\section{Experimental}

\subsection{Materials}

Two different types of post-consumer plastic, PET and LDPE were chosen to verify the operation of equipment. Two different kinds of feedstock were utilised: (i) dry blended mixture of virgin resins of PET/LDPE in 70/30 wt/wt ratio, where PET was Arnite ${ }^{\circledR}$ D00 301 produced by DSM (density $1.34 \mathrm{~g} / \mathrm{cm}^{3}, T_{m}=250^{\circ} \mathrm{C}$ ) and LDPE was TIPOLEN FA 244-51 (density 0.92 $\mathrm{g} / \mathrm{cm}^{3}$, MFI $0.28 \mathrm{~g} / 10 \mathrm{~min}$ ) from TVK Nyrt (Hungary); and (ii) prefabricated polymer blends in a melted state from the same primary components. The total weight of each mixture and blend was $70 \mathrm{~g}$.

\subsection{Equipment}

The primary PET/PE blends were prepared in an internal mixer (Brabender Plasti-Corder PL2000), where the barrel temperature was $250^{\circ} \mathrm{C}$, melt-blending time was $10 \mathrm{~min}$ and the mixing speed was $20 \mathrm{rpm}$. The melt temperature $T_{m}$ of the resins and blends was determined by differential scanning calorimetry, using DSC Q2000 in a nitrogen atmosphere. Every sample (4-6 mg) was sealed in aluminium pans and heated from -40 to $340^{\circ} \mathrm{C}$, at a heating rate of $20^{\circ} \mathrm{C} / \mathrm{min}$. The complex viscosities were recorded using an AR2000 rheometer (TA Instruments) in plate-plate configuration, where the discs of $25 \mathrm{~mm}$ in diameter were obtained by compression-moulding blends for 5 minutes at $290^{\circ} \mathrm{C}$. A Nabertherm L9/11/C6 furnace was used as the heat source. The samples were prepared by using Struers LaboPol-5 automatic polishing machine to $1 \mu \mathrm{m}$ accuracy.

An Olympus BX 51M optical microscope was used to determine the efficiency of separation, and a JEOL JSM 6380LA scanning electron microscopy (SEM) was used at an acceleration voltage of $20 \mathrm{kV}$, to study the morphological structures of the blends. The Raman spectra were collected using a Horiba Jobin-Yvon LabRAM system, coupled with an external $532 \mathrm{~nm} \mathrm{Nd-}$ YAG laser source and an Olympus BX-40 optical microscope. The Raman spectra of the ingredient were established with a 10x objective, using an acquisition time of 5 to $10 \mathrm{~s}$, and accumulating five measured spectra at a time. The analysis software was NGS Labspec v5.76.

\subsection{Description of the developed separation equipment}


Fig. 2 shows the schematic representation of the constructed horizontal separator, in which the separation occurred in a melted state by centrifugal forces. The shaft with a diameter of $17 \mathrm{~mm}$ rotates in the bearings. The separation tank can be found on the shaft, which consists of two closing cups and a cylinder (75 $\mathrm{mm}$ inner diameter and $3.5 \mathrm{~mm}$ thickness). The closing is realized with tight-fitting and spring tension. The closing cups can be removed from the cylinder so the pre-defined compositional ratio of the materials can be dosed to the tank, and after the process, the solidified structure can also be removed with the same method. The other end of the shaft is grooved, thus it can be rotated with AC motors.

The separation tank spins together with the shaft. Therefore, the shear forces are small under the steady state condition of the separation. The separation of the partially or fully melted blends at low shear rates is caused because of the three apparent forces, (i) the centrifugal force; (ii) the Coriolis-force; and (iii) the Euler-force. The latter can be negligible, because it only affects the separation of the phases in the initial angular acceleration stage. It is shown in Eq. (1) that the centripetal acceleration of the particles only depends on the applied speed of the equipment, as well as the distance of the particle from the shaft. The centripetal acceleration is 30 times higher than the acceleration of gravity near the shaft and more than 100 times at the walls of the cylinder, at the applied rotation speed of $1800 \mathrm{rpm}$ (Fig 3.). So the influence of gravity factor is negligible. The efficiency of the separation also depends on the size and shape of the particulars, and the applied melting temperature. The latter influences the complex viscosity and the specific volume of the materials.

The constructed separator, in which the sample is also situated, was heated in a furnace. The separation process occurred at different temperatures. The heating was 60 minutes for every sample, after $10 \mathrm{~min}$ spinning was applied (keeping the same temperature) to dry blended mixtures of PET/LDPE 70/30 wt/wt, and 20 min spinning of melted blends of PET/LDPE 70/30 $\mathrm{wt} / \mathrm{wt}$. The spinning of the shaft was $1800 \mathrm{~min}^{-1}$ in all cases at rotation stage. Finally, the shapefixing of the samples was realized in a cold aqueous medium, also under spinning. After the separation process, the samples were prepared by polishing machine.

\section{Results and discussion}

\subsection{DSC measurement for analysing thermal behaviour}

The DSC plots of the primary pellets and the blends in the heating runs are shown in Fig. 4. As can be seen, the melting points $\left(T_{m}\right)$ of PET and LDPE granules are $252^{\circ} \mathrm{C}$ and $110^{\circ} \mathrm{C}$, and the melting ranges are 235 to $260^{\circ} \mathrm{C}$ and 90 to $115^{\circ} \mathrm{C}$, respectively. A double melting peak can be seen at $70 / 30 \mathrm{wt} / \mathrm{wt}$ blends of PET/LDPE $\left(252^{\circ} \mathrm{C}, 108^{\circ} \mathrm{C}\right)$, respectively. After the results of DSC measurement, the separation process occurred through the mixtures and the blends at different temperatures at and above the melting peak of the PET.

\subsection{Morphology analysis of the blend}

In a melted state the 70/30 PET/LDPE blend showed a dispersed morphology, where the spherical-shaped particles were spread in the matrix layer (Fig. 5). The particle sizes show a wide range distribution from 10 to $100 \mu \mathrm{m}$. As expected (Cardinaud and McNally, 2013), LDPE 
and PET are immiscible with each other, and the interfacial adhesion between the particles is very poor. Indeed, the dispersed particles debonded from the matrix are definitely loose.

\subsection{Viscosity measurement of the applied polymers}

Fig. 4 represents the complex viscosities of LDPE (upper diamond points), PET (lower square points) and the 70/30 wt/wt PET/LDPE blends (middle triangle points) as a function of shear rate at a temperature of $290^{\circ} \mathrm{C}$. All the samples showed shear thinning behaviour over the investigated range of the shear rate. The complex viscosity of LDPE is found to be higher than PET at $290^{\circ} \mathrm{C}$, as a result of the used LDPE made for film blowing, LDPE is less sensitive to temperature changes. At a small shear rate, the two components determined the viscosity of the blend, but at a higher shear rate, the rheological property of the blend matches with the characteristics of the PET curve. The separation process occurred at small shear rates.

\subsection{Separation results of PET/LDPE mixtures}

At $250^{\circ} \mathrm{C}$ the separation of the phases of the dry blended mixtures from granules was not successful. Although the LDPE phases were in a melted state, PET phases remained in a completely solid state (Fig. 7(a)). Based on the DSC curves (Fig. 4.) the PET phases should have at least partly melted at $250^{\circ} \mathrm{C}$. This probably occurs because LDPE, which was melting first at a lower temperature (around $110^{\circ} \mathrm{C}$ ), enriched around the wall of the cylinder and functioned as an insulator during heating $\left(60 \mathrm{~min}, 250^{\circ} \mathrm{C}\right)$, so PET phases could not melt at the value of the melting peak of the PET. It should be noted, that in the case of the internal mixing, the PET particles melted at $250^{\circ} \mathrm{C}$ because of the higher shear forces. So it can be stated that, in the separation process, the shear forces are much smaller than inside the internal mixer.

With the chosen processing parameters at lower temperature $\left(250^{\circ} \mathrm{C}\right)$, where PET has the melting peak (Fig. 4), the phase separation can-not be achieved. The enrichment of the solid state PET phase was not implemented near the wall of the cylinder. The reasons for this problem could be as follows: (i) PET phases had higher viscosity because the PET did not melt, so the droplets could not migrate towards the wall of the cylinder; (ii) the applied speed was low during the separation process; and (iii) the spinning time (10 min) and/or the heating time (60 min) was not enough. Nevertheless, a much longer heating time is not recommended because of the degradation of the polymers.

In order to melt the PET granules a higher temperature was used, $290^{\circ} \mathrm{C}$; more than $30^{\circ} \mathrm{C}$ of the upper melting limit of PET. The separation of the phases was successful. The full phase separation is clearly visible in Fig. 8(a), where the LDPE and PET parts were completely melted. Owing to the influence of the centrifugal and Coriolis force, the molten droplets of PET, which have a higher density than LDPE, can migrate towards the wall of the cylinder, while the LDPE phases move towards the shaft during the spinning of the separation process.

According to the microscopy images, the outer layer of the sample consists only of PET phases (Fig. 8(b)), while the inner layer only contains LDPE (Fig 8(c)). Between the zones a sharp transition boundary can be observed (Fig. 8(d)), where a large number of cracks can also be discovered, as a result of the different shrinkage of the polymers. 
The DSC results from the outer and inner layers of the samples were compared with DSC curves of the virgin granules, to establish the purity of the two layers. The particles were sampled from the separated disc, which was produced at $290^{\circ} \mathrm{C}$. As seen in Fig. 9, the sampled particles show similar curves to the reference polymers during the heating process. Examining the curves, it can be stated that no contaminants can be detected either at the outer or the inner layer. The obtained results of the DSC measurement are in agreement with the results of the microscopy.

It can be stated, however, that the PET did not melt during the separation process at a lower temperature, and the separation did not take place between the phases of the polymer mixtures. Therefore, all the phases must be in a completely melted condition in order to separate the polymers from each other successfully in a melted state.

\subsection{Separation results of PET/LDPE blends}

Despite the fact that, under the same processing parameter as used at polymer mixture (10 minutes spinning time after the 60 minutes heating at $290^{\circ} \mathrm{C}$ ), it was found that the separation of the internal mixed, prefabricated polymer blends into individual homogeny phase was not repeatable. Although a few enrichment phases of the same components on the discs were found, the separation of the two components cannot be confirmed.

The separation of the blend at $290^{\circ} \mathrm{C}$, during 20 minutes spinning time, also ended with only partial success (Fig 10(a)). Regardless of the fact that both components were fully melted, the separation of the phase did not occur. At the outer edge of the disc a clearly homogeneous PET phase had begun to develop (Fig. 10(b)), but the thickness of this phase was very varied. The optical images show that inside the LDPE-rich inner layer, PET components can also be found in large quantities (Figs. 10(c)-(d)). A sharp transition boundary zone can not be seen in the sample. Therefore, the sample can only be divided into two major parts: (i) outer zone with thickness 0.5-1.5 mm, which contains mainly PET phases; and (ii) inner zone, which was 6 to 9 times thicker than the outer one, and contains LDPE and also PET in significant amounts. This result shows that the processing temperature, which was enough for separating the components of the mixture into a homogenous and continuous phase, is not sufficient for polymer blends. The chosen parameters during the heating and spinning stages are inadequate to separate the polymers into individual phases, based on their difference in density. With this result, it can be demonstrated that not only does the difference in density have an influence on the separation process, but also the interfacial adhesion of the polymer parts. If the size of the separated phase is smaller a larger specific surface is implemented, and thereby more adhesion can be gained between the two polymer parts, which need further energy to separate fractions into pure fragments.

At $310^{\circ} \mathrm{C}$, the separation of the phases of the blend was successful, and a nearly full phase separation is visible in Fig. 11(a). The higher density PET migrated towards the wall of the cylinder, while the lower density LDPE phases moved towards the shaft, during the spinning at the separation process. At $310^{\circ} \mathrm{C}$, the polymers can be characterized with lower viscosity, and the size of the melted droplets are maybe smaller, owing to higher flow properties than at $290^{\circ} \mathrm{C}$. Thereby, the droplets can separate more easily based on their differences in density. This 
phenomenon can have the result that, at the wall of the cylinder only PET can be found, while at the inner layer of the sample only LDPE.

According to the microscopy images, the outer layer of the sample consists of almost only PET phases (Fig. 11(b)), while the inner layer contains LDPE in particular, but also a small amount of the phase of PET. Fig 11(c) shows a PET-enriched region in the inner layer. Between the zones an elongated transition boundary can be observed, with a thickness of $0.5 \mathrm{~mm}$, which is 8 to 9 times thinner that the pure PET layer (Fig. 11(d)).

The DSC results from the outer and inner zones of the samples were compared with DSC curves of the virgin granules, to verify the purity of the layers qualitatively (Fig. 12). The particles were sampled from the separated disc, which was produced at $310^{\circ} \mathrm{C}$. The melting peaks of the reference of LDPE and PET were $110^{\circ} \mathrm{C}$ and $252^{\circ} \mathrm{C}$, respectively. The samples from the outer layer showed the expected melting range from $240^{\circ} \mathrm{C}$ to $260^{\circ} \mathrm{C}$ for the PET, but a very slight new endothermic peak appeared at $108^{\circ} \mathrm{C}$, which means the presence of a small amount of the LDPE in the outer layer. The samples from the inner layer also show two melting peaks, at $108^{\circ} \mathrm{C}(\mathrm{LDPE})$ and $252^{\circ} \mathrm{C}(\mathrm{PET})$. But in this case the presence of the second endothermic peak of PET is more characteristic, which assumes that the inner layer contains more contaminants. But the DSC result indicates that the proportion of crystalline PET parts is significantly lower than the LDPE. The area under the melting zone of LDPE was seven times higher than PET $(42.2 \mathrm{~J} / \mathrm{g}, 5.92 \mathrm{~J} / \mathrm{g}$, respectively). The obtained results of the DSC measurement are in a good agreement with the results of the microscopy.

Finally the purity of the fractions was analysed with Raman spectroscopy. The samples are from the outer (Fig. 13(a)) and inner (Fig 13(b)) layers, where the upper curves represent the spectra of the sample: 'middle' means the reference of PET, and 'lower' is the spectrum of the reference LDPE. The degradation can be deducted from each sample, but in both cases the characteristic peaks is clearly visible. The presence of LDPE was not detectable from the spectrum of the sample from the outer zone. Conversely, the spectrum of the sample from the inner zone, characteristic peaks of PET has been released.

Summarizing the results of this research; the separation was successful overall. The results of the separation of polymer mixtures at $290^{\circ} \mathrm{C}$ show two neat separated fractions, with no detectable contamination. The monitoring measurement (DSC, optical and Raman spectroscopy) after the separation of polymer blends at higher temperatures indicates two high purity fractions in the samples, while the outer zone consists of PET and LDPE forms mainly in the inner layer in both cases. It can be assumed that the difference between the viscosities of the components has also a significant role for the separation. Polymers with lower viscosity can be characterized by greater flow ability and major Brownian motion of the segments.

Based on the results of the separation of prefabricated polymer blends it was found that, in this case, it was needed to administer more energy in the form of heat. This is presumably a result of the fact that blends have a finer dispersed morphology by three orders of magnitude than the dry blended mixtures. Thus, the different components can be separated from each other. After that, in the outer zone the PET and inner layer the LDPE could coalesce into nearly pure and homogenous parts, but a higher temperature and longer spinning time were required. The 
increase of the speed, presumably as a result of the purity of the separated fractions, could be improved further, owing to the larger acting forces.

\section{Conclusion}

In this research a novel separation device was built and tested, to separate polymers from each other in high purity. The equipment utilizes centrifugal force to separate the fractions in a melted state. After the separation in the centrifugal force field, owing to the difference of density of PET and LDPE, two high purity fractions were established in the samples, where the outer zone consisted of PET, and mainly LDPE formed the inner layer. The results show that the developed equipment is suitable not only for separating dry blended mixtures of PET/LDPE into pure components again, but also for prefabricated polymer blends. Hence, this was the first time it was possible to separate two different plastics into neat fractions in a melted state with the developed equipment. This polymer blend separation process is completely different, faster and more environmentally friendly technology than using solvent agents. With this process it may become possible, during the recycling process, to recover the pure polymer substances from products, which were manufactured from several polymer substances at the same time.

Based on the experiments it can be stated that the applied temperature and the speed had a significant impact on separation. In the case of polymer blends a higher separation temperature and longer spinning time were required, which can probably be explained by the decrease of the size of the particles to three orders of magnitude compared to the granules. In other words, a much finer distribution of morphology was formed at polymer blends, and it seems more time is required for the coalescence of the same phases to form the separated layers.

Examining how the coalescence of the droplets occurred under centrifugal force should be the subject of future research. Thereby it would be an option to classify a multi-component mixture of waste according to which component appears in which proportion in the waste stream.

\section{Acknowledgement}

This research was realized in the framework of TÁMOP 4.2.4. A/1-11-1-2012-0001 "National Excellence Program - Elaborating and operating an inland student and researcher personal support system". The project was subsidized by the European Union and co-financed by the European Social Fund. The infrastructure of the research project was supported by the Hungarian Scientific Research Fund (OTKA K109224).

\section{References}

Alter, H., 2005. The recovery of plastics from waste with reference to froth flotation. Resour. Conserv. Recy. 43, 119-132.

Arvanitoyannis, I.S., Bosnea, L.A., 2001. Recycling of polymeric materials used for food packaging: current status and perspectives. Food. Rev. Int. 17, 291-346.

Bakker, E.J., Rem, P.C., Fraunholcz, N., 2009. Upgrading mixed polyolefin waste with magnetic density separation. Waste Manage. 29, 1712-1717. 
Batalović, V., 2011. Centrifugal separator, the new technical solution, application in mineral processing. Int. J. Miner. Process. 100, 86-95.

Bezati, F., Froelich, D., Massardier, V., Maris, E., 2011. Addition of X-ray fluorescent tracers into polymers, new technology for automatic sorting of plastics: Proposal for selecting some relevant tracers. Resour. Conserv. Recy. 55, 1214-1221.

Bodzay, B., Marosfoi, B.B., Igricz, T., Bocz, K., Marosi, Gy., 2009. Polymer degradation studies using laser pyrolysis-FTIR microanalysis. J. Anal. Appl. Pyrol. 85, 313-320.

Burat, F., Güney, A., Kangal, M.O., 2009. Selective separation of virgin and post-consumer polymers (PET and PVC) by flotation method. Waste Manage. 29, 1807-1813.

Cardinaud, R., McNally, T., 2013. Localization of MWCNTs in PET/LDPE blends. Eur. Polym. J. 49, 1287-1297.

Carvalho, M.T., Agante, E., Durao, F., 2007. Recovery of PET from packaging plastics mixtures by wet shaking table. Waste Manage. 27, 1747-1754.

Fávaro, S.L., Freitas, A.R., Ganzerli, T.A., Pereira, A.G.B., Cardozo, A.L., Baron, O., Muniz, E.C., Girotto, E.M., Radovanovic, E., 2013. PET and aluminium recycling from multilayer food packaging using supercritical ethanol. J. Supercrit. Fluid. 75, 138-143.

Gent, M.R., Menendez, M., Torano, J., Isidro, D., Torno, S., 2009. Cylinder cyclone (Larcodems) density media separation of plastic waste. Waste Manage. 29, 1819-1827.

Gent, M.R., Menendez, M., Torano, J., Torno, S., 2011. Optimization of the recovery of plastics for recycling by density media separation cyclones. Resour. Conserv. Recy. 55, 472-482.

Giancola, G., Lehman, R.L., Idol, J.D., 2012. Melt processing and domain morphology of PMMA/HDPE polymer blends prepared from powder precursors. Powder Technol. 218, 18-22.

Gondal, M.A., Siddiqui, M.N., 2007. Identification of different kinds of plastics using laserinduced breakdown spectroscopy for waste management. J. Environ. Sci. Heal. A. 42, 1989-1997.

Kim, T-H., Hwang, H., Gorkin, R., Madou, M., Cho, Y-K., 2013. Geometry effects on blood separation rate on a rotating disc. Sensor. Actuat. B-Chem. 178, 648- 655.

Kraai, G.N., Schuur, B., van Zwol, F., van de Bovenkamp, H.H., Heeres, H.J., 2009. Novel highly integrated biodiesel production technology in a centrifugal contactor separator device. Chem. Eng. J. 154, 384-389.

Matsusaka, S., Maruyama, H., Matsuyama, T., Ghadiri, M., 2010. Triboelectric charging of powders: a review. Chem. Eng. Sci. 65, 5781-5807.

Pongstabodee, S., Kunachitpimol, N., Damronglerd, S., 2008. Combination of three-stage sinkfloat method and selective flotation technique for separation of mixed post-consumer plastic waste. Waste Manage. 28, 475-483.

Ramesh, S., Leen, K.H., Kumutha, K., Arof, A.K., 2007. FTIR studies of PVC/PMMA blend based polymer electrolytes. Spectrochim. Acta A. 66, 1237-1242. 
Sadat-Shojai, M., Bakhshandeh, G.-R., 2011. Recycling of PVC wastes. Polym. Degrad. Stabil. 96, 401-415.

Serranti, S., Gargiulo, A., Bonifazi, G., 2011. Characterization of post-consumer polyolefin wastes by hyperspectral imaging for quality control in recycling processes. Waste Manage. 31, 2217-2227.

Strixner, T., Kulozik, U., 2013. Continuous centrifugal fractionation of egg yolk granules and plasma constituents influenced by process conditions and product characteristics. J. Food Eng. 117, 89-98.

Super, M.S., Enick, R.M., Beckman, E.J., 1993. Density-based separation of thermoplastics found in the post-consumer waste stream. Resour. Conserv. Recy. 9, 75-88.

Takoungsakdakun, T., Pongstabodee, S., 2007. Separation of mixed postconsumer PET-POMPVC plastic waste using selective flotation. Sep. Purif. Technol. 54, 248-252.

Vajna, B., Bodzay, B., Toldy, A., Farkas, I., Igricz, T., Marosi, Gy., 2012. Analysis of car shredder polymer waste with Raman mapping and chemometrics. Express. Polym. Lett. 6, 107119.

Vermeulen, I., Van Caneghem, J., Block, C., Baeyens, J., Vandecasteele, C., 2011. Automotive shredder residue (ASR): Reviewing its production from end-of-life vehicles (ELVs) and its recycling, energy or chemicals' valorisation. J. Hazard. Mater. 190, 8-27.

Wang, L., Shi, J., Liu, L., Secret, E., Chen, Y., 2011. Fabrication of polymer fiber scaffolds by centrifugal spinning for cell culture studies. Microelectron. Eng. 88, 1718-1721.

Wills, B.A., 1997. Mineral Processing Technology. Pergamon Press, Oxford.

Wu, G., Li, J., Xu, Z., 2013. Triboelectrostatic separation for granular plastic waste recycling: A review. Waste Manage. 33, 585-597.

Yamamoto, T., Watanabe, N., Fukui, K., Yoshida, H., 2009. Effect of inner structure of centrifugal separator on particle classification performance. Powder Technol. 192, 268-272.

Zhang, C., Huang, K., Yu, P., Liu, H., 2012. Sugaring-out three-liquid-phase extraction and one-step separation of Pt(IV), Pd(II) and Rh(III). Sep. Purif. Technol. 87, 127-134. 


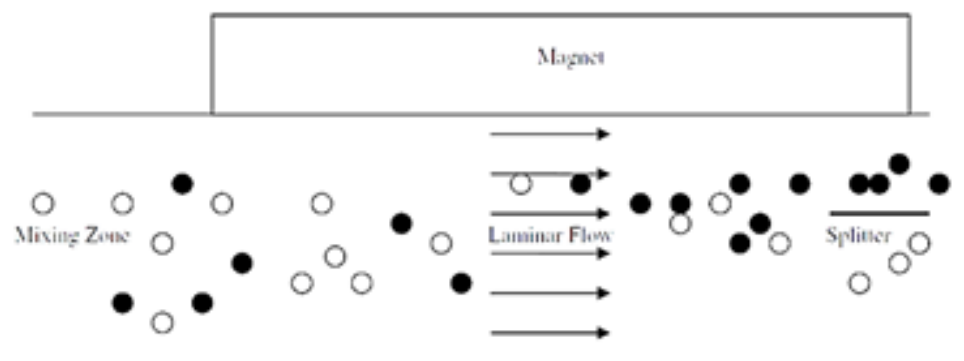

Fig. 1. A schematic drawing of the experiment with an IMDS • PP; ○ PE particles [Bakker et al., 2009] 


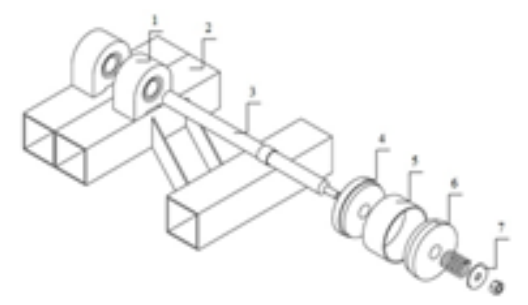

Fig. 2. A schematic drawing of the constructed horizontal separator: (1) bearing housing; (2) welded frame; (3) shaft of $17 \mathrm{~mm}$ diameter; (4) bottom closing cup; (5) cylinder of $75 \mathrm{~mm}$ inner diameter; (6) upper closing cup; (7) clamping anchors 


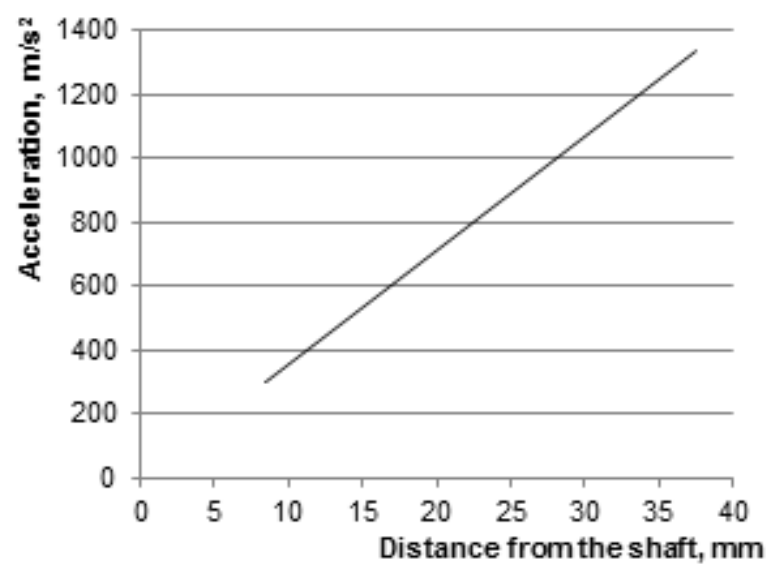

Fig. 3. The affected centripetal acceleration depending on the distance from the shaft 


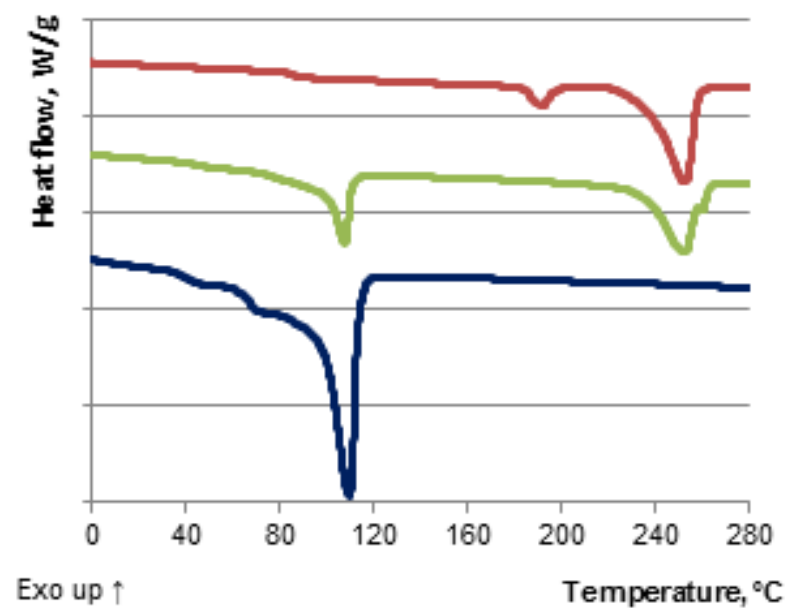

Fig. 4. DSC heating curves at $20^{\circ} \mathrm{C} / \mathrm{min}$ of PET, LDPE and their blends, - PET; $-70 / 30$ PET/LDPE; - LDPE 


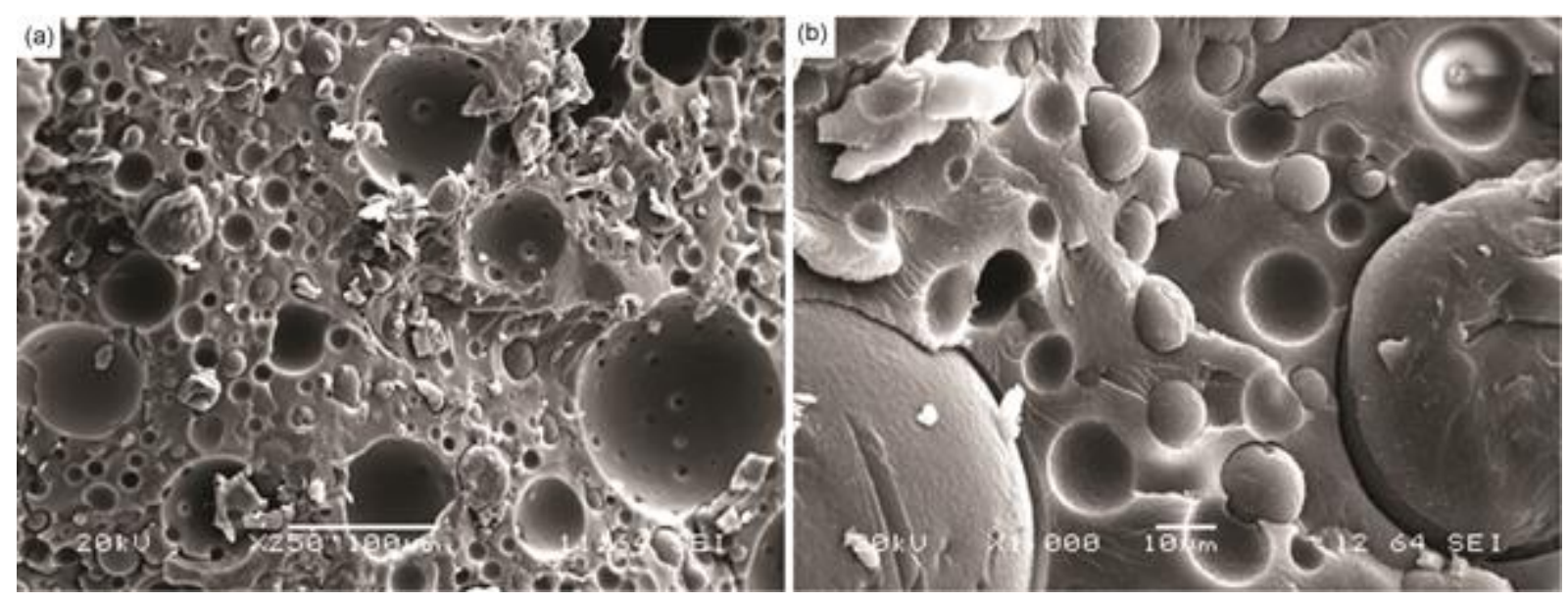

Fig. 5. SEM images from the PET/PE 70/30 prefabricated material of the blends: (a) x250; (b) x1000 magnification 


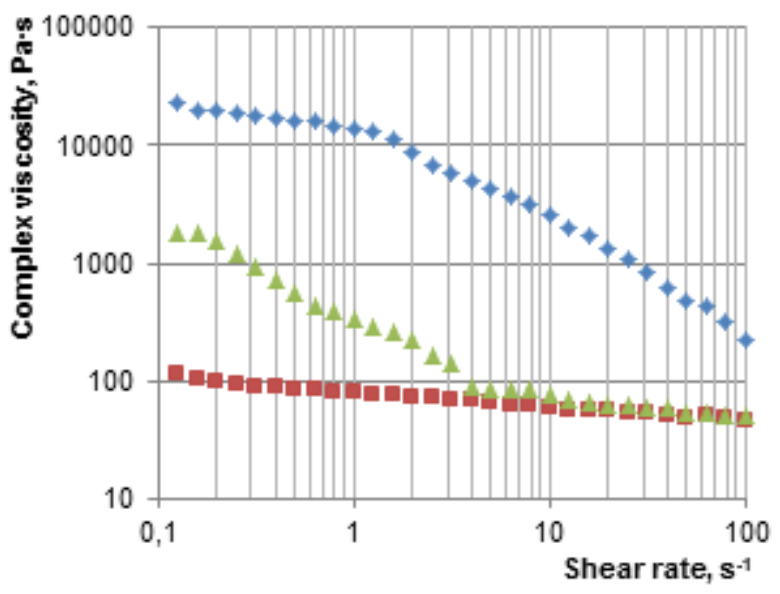

Fig. 6. Viscosity of ( $\diamond$ LDPE, ( $\square$ ) PET and ( $\triangle$ ) 70/30 PET/LDPE blend at $290^{\circ} \mathrm{C}$ 

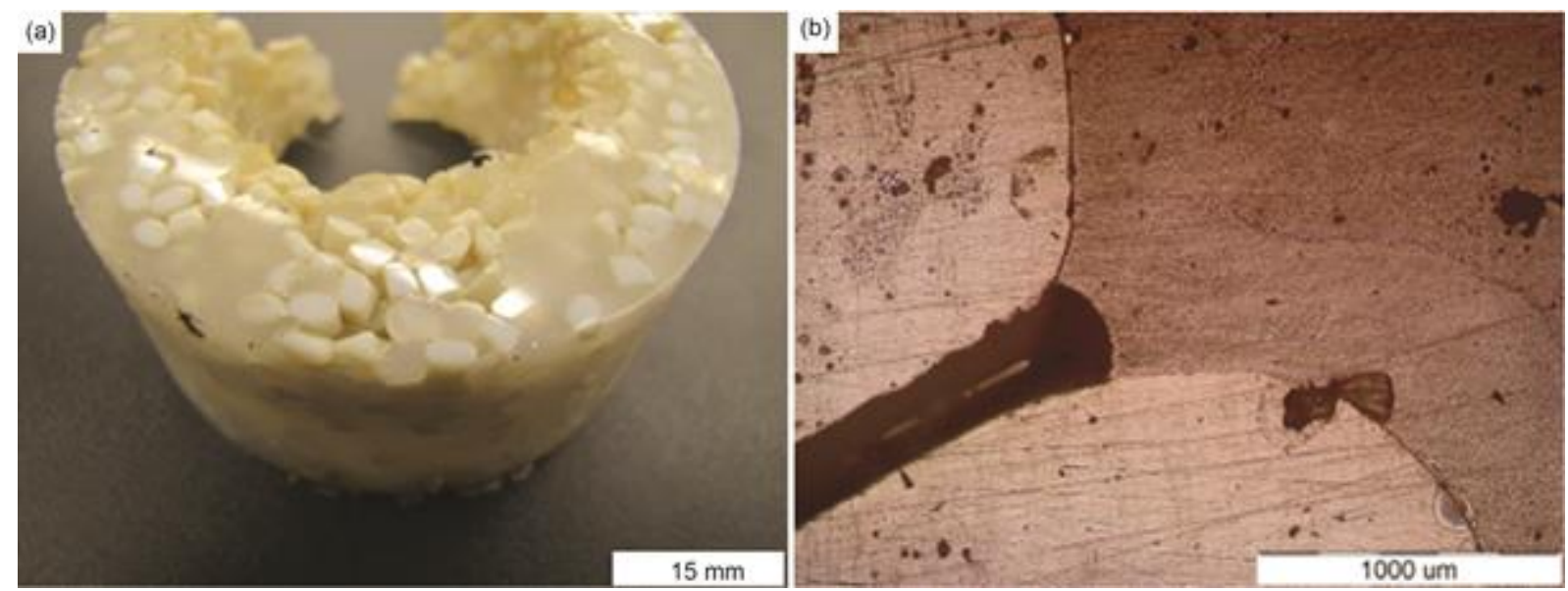

Fig. 7. Separation result at $250^{\circ} \mathrm{C}$ : (a) total cross section of the sample; (b) optical microscopy of PET and LDPE parts at 5x magnification 


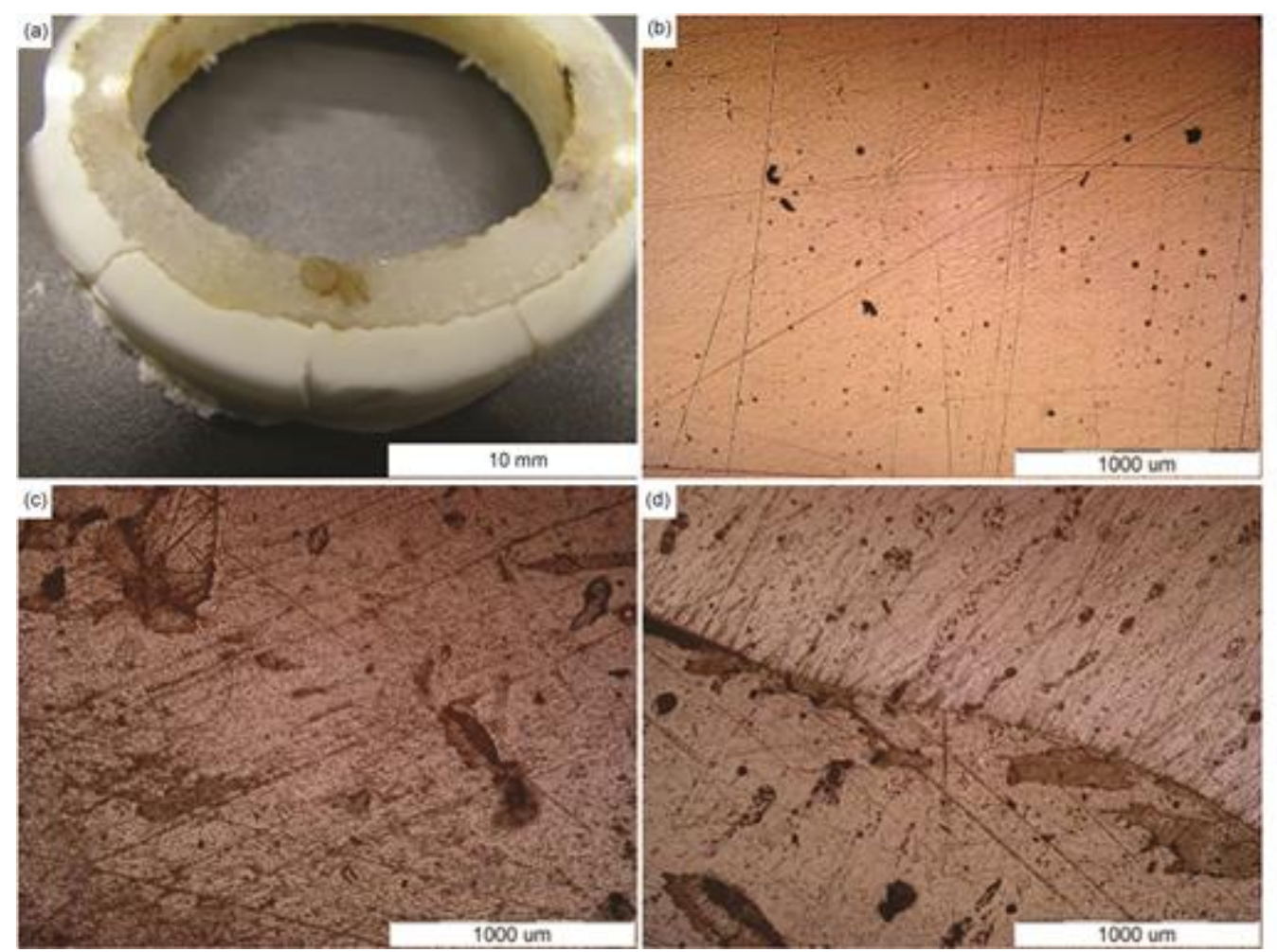

Fig. 8. Separation result at $290^{\circ} \mathrm{C}$ of $70 / 30$ PET/LDPE mixture: (a) total cross section of the sample; (b) outer layer; (c) inner layer; (d) sharp boundary zone 


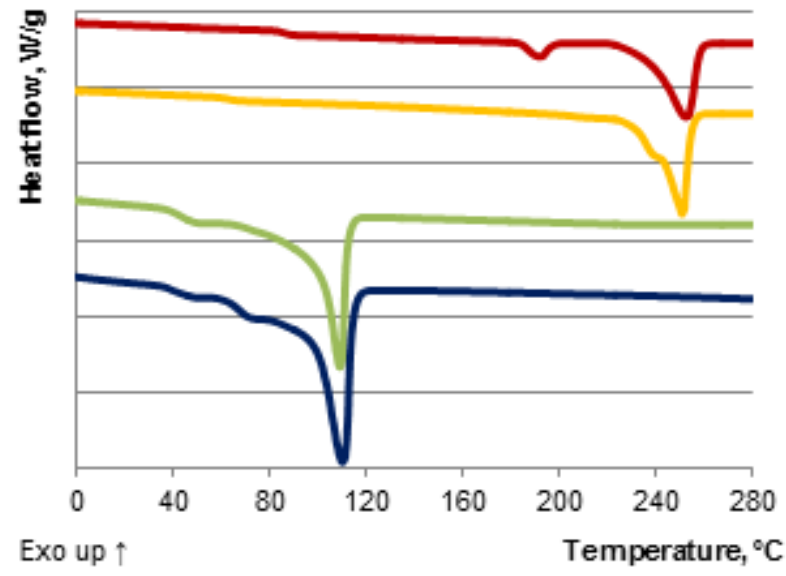

Fig. 9. DSC analysis of the sample (mixture, $290^{\circ} \mathrm{C}$ ), - Ref PET; - Outer layer sample; Inner layer sample; - Ref LDPE 

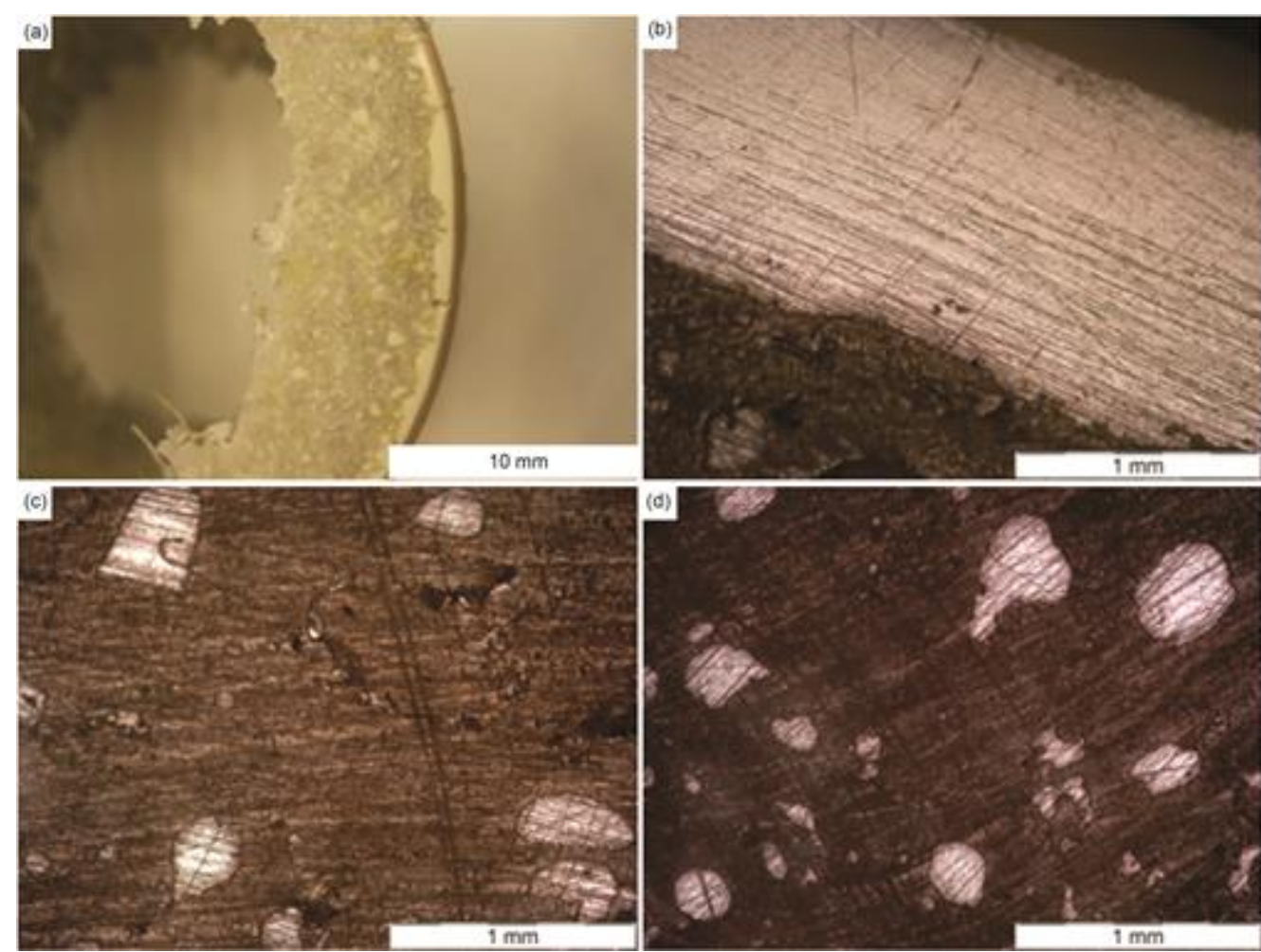

Fig. 10. Separation result at $290^{\circ} \mathrm{C}$ of $70 / 30$ PET/LDPE blend: (a) total cross section of the sample; (b) outer layer; (c)-(d) inner layer 


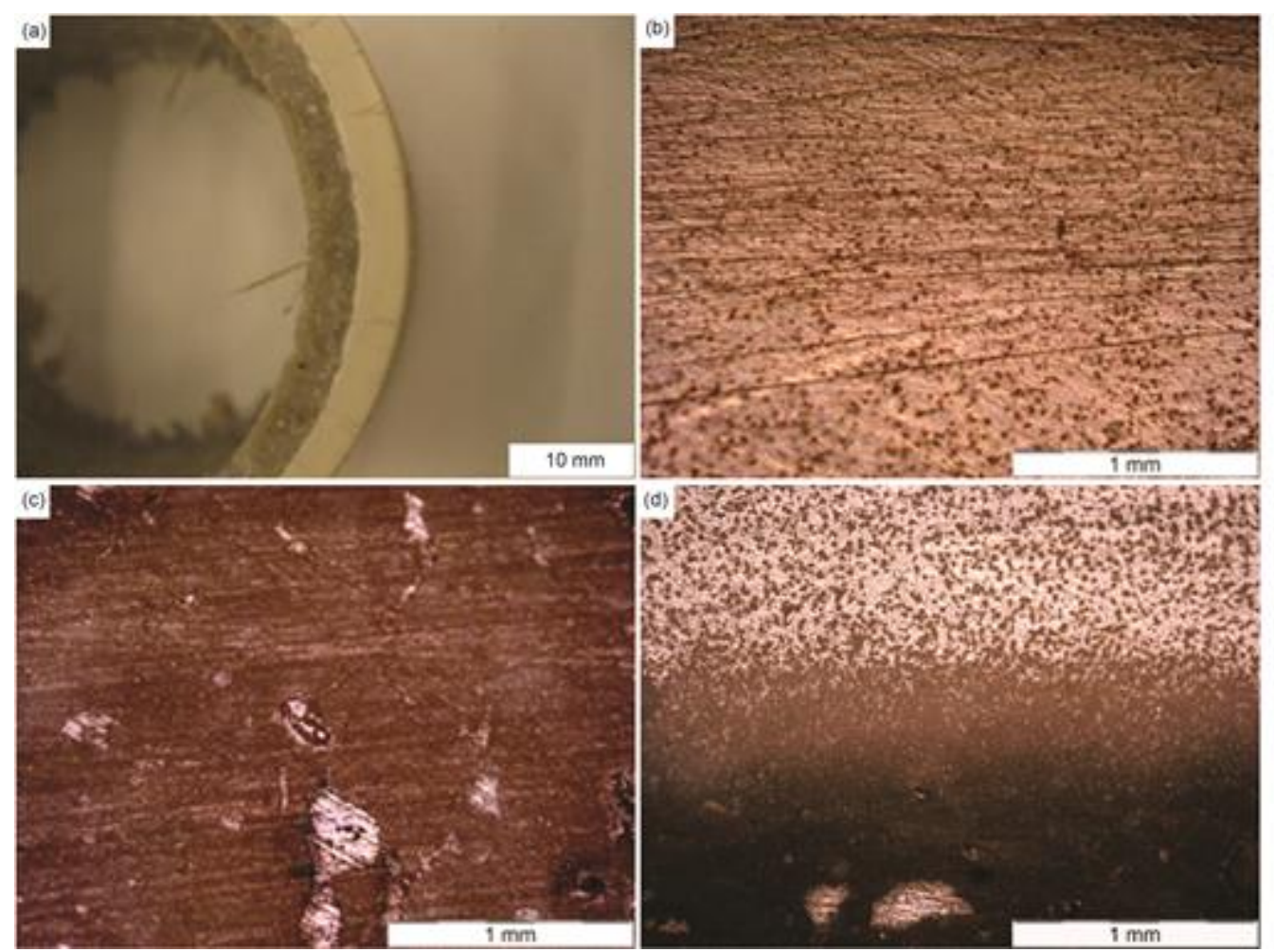

Fig. 11. Separation result at $310^{\circ} \mathrm{C}$ of $70 / 30$ PET/LDPE blend: (a) total cross section of the sample; (b) outer layer; (c) inner layer; (d) boundary zone of the separation 


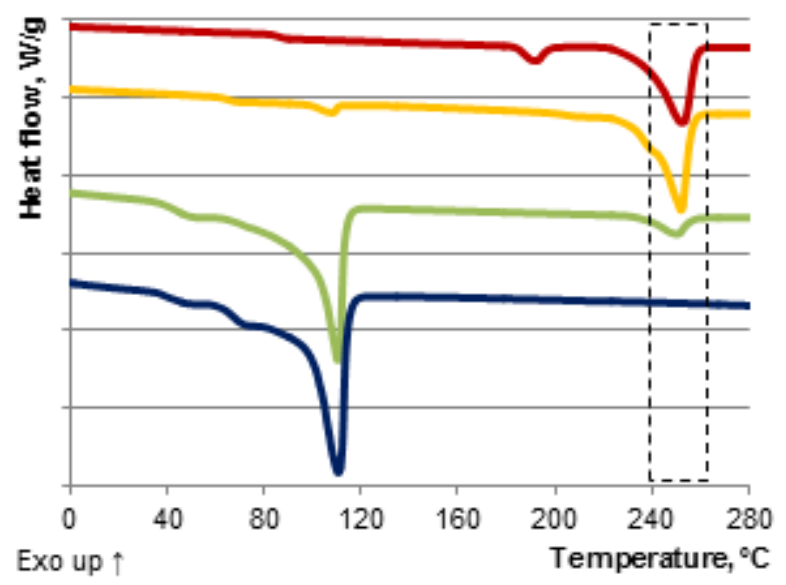

Fig. 12. DSC analysis of the sample (blend, $310^{\circ} \mathrm{C}$ ), - Ref PET; - Outer layer sample; - Inner layer sample; - Ref LDPE 

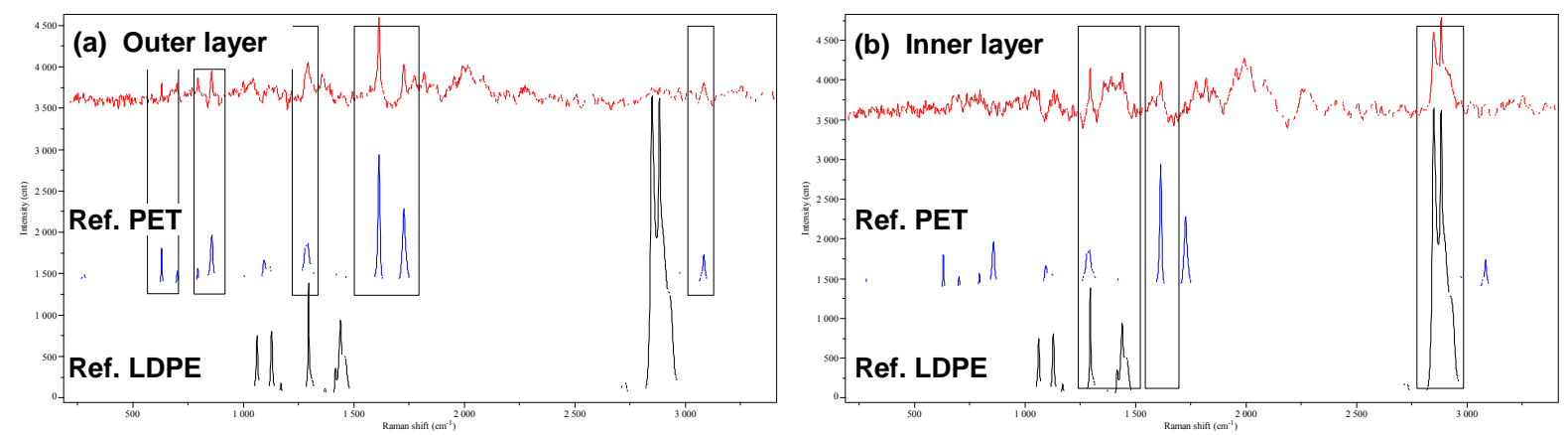

Fig. 13. Raman spectra of particles from (a) the outer, and (b) the inner layer of the sample $\left(310^{\circ} \mathrm{C}\right)$ 